\title{
Syndrome d'activation macrophagique chez un patient greffé rénal
}

\author{
Haemophagocytic Lymphohistiocytosis in a Patient with a Kidney Transplant
}

\author{
L. Sindzingre $\cdot$ A. Elezi $\cdot$ O. Peyrony \\ Reçu le 1 juillet 2019; accepté le 2 octobre 2019 \\ (C) SFMU et Lavoisier SAS 2019
}

\section{Introduction}

Le syndrome d'activation macrophagique (SAM), ou syndrome d'hémophagocytose lymphohistiocytaire, résulte d'une hyperproduction de cytokines et d'une stimulation inappropriée des macrophages survenant le plus souvent dans un contexte d'immunosuppression, et dont le facteur déclenchant est fréquemment infectieux ou néoplasique. Le tableau clinicobiologique associe fièvre, splénomégalie, cytopénies, cytolyse hépatique, hypertriglycéridémie et hyperferritinémie, auquel s'ajoute une hémophagocytose intramédullaire. La gravité de ce syndrome est liée au risque d'apparition de défaillances d'organes. Le diagnostic est souvent difficile, car les symptômes sont peu spécifiques et peuvent mimer ceux d'un sepsis ; il est d'autant plus difficile que ce syndrome peut en être la conséquence [1]. Cependant, si ce diagnostic est évoqué, certains éléments biologiques tels que l'hyperferritinémie et l'hypertriglycéridémie peuvent permettre au clinicien d'en conforter l'hypothèse. Évoquer un SAM devant un tableau compatible chez un patient immunodéprimé dès sa prise en charge aux urgences permet d'en améliorer le pronostic, en recherchant d'emblée son facteur déclenchant et en initiant rapidement une prise en charge thérapeutique adaptée. Nous rapportons ici l'observation d'un cas de SAM chez un patient greffé rénal.

\section{Observation}

Un homme de 70 ans consulte aux urgences pour des lésions cutanéomuqueuses fébriles (Fig. 1) évoluant depuis deux semaines, sans amélioration malgré la prise d'antibiotiques (amoxicilline-acide clavulanique) depuis sept jours, ainsi qu'une altération franche de l'état général depuis deux jours.

L. Sindzingre $\cdot$ A. Elezi $\cdot$ O. Peyrony $(\bowtie)$

Service des urgences, hôpital Saint-Louis,

Assistance publique-Hôpitaux de Paris,

11, avenue Claude-Vellefaux, F-75010 Paris, France

e-mail : o.peyrony@hotmail.fr
Dans ses antécédents, on note une cardiopathie ischémique, une hypertension artérielle, un diabète de type 2 et une néphropathie d'étiologie inconnue pour laquelle il a été transplanté un an auparavant. Lors de la greffe, il existait un mismatch pour le cytomégalovirus (CMV). Notons également que le patient a reçu un traitement d'induction de déplétion des lymphocytes $\mathrm{T}$ le jour de la greffe. Suite à la greffe rénale, il prend un traitement immunosuppresseur par du mycophénolate mofétil, remplacé par de l'azathioprine une semaine avant, et de la ciclosporine. Le patient présente depuis la greffe une dysfonction chronique du greffon avec, un an auparavant, une créatinine à $190 \mu \mathrm{mol} / \mathrm{l}$, soit un débit de filtration glomérulaire (DFG) estimé à $30 \mathrm{ml} / \mathrm{min}$ par $1,73 \mathrm{~m}^{2}$ et une protéinurie glomérulaire modérée à sévère à environ $1,3 \mathrm{~g} / \mathrm{j}$.

Aux urgences, la température est à $38,4{ }^{\circ} \mathrm{C}$. L'examen clinique montre des ulcérations de la lèvre, de la langue et de l'avant-bras, de quelques centimètres, nécrotiques et malodorantes, ainsi qu'une splénomégalie sans hépatomégalie associée à une adénopathie centimétrique axillaire. La biologie objective une pancytopénie (anémie à $9,1 \mathrm{~g} / \mathrm{dl}$ arégénérative (14 000 réticulocytes $\left./ \mathrm{mm}^{3}\right)$, leucopénie à $830 / \mathrm{mm}^{3}$, dont 440 neutrophiles, et thrombopénie à $116000 / \mathrm{mm}^{3}$ ), sans schizocytes circulants ni stigmate d'hémolyse, une aggravation de la dysfonction chronique du greffon (créatinine à $245 \mu \mathrm{mol} / \mathrm{l}$, avec un DFG estimé à $22 \mathrm{ml} / \mathrm{min}$ par $1,73 \mathrm{~m}^{2}$ ), une cholestase à $4 \mathrm{~N}$ sans élévation de la bilirubine ni cytolyse, un taux de lactate déshydrogénase (LDH) à $2 \mathrm{~N}$, un taux de prothrombine à $90 \%$ et un fibrinogène à $5,36 \mathrm{~g} / 1$ (N 2 à $4 \mathrm{~g} / \mathrm{l}$ ). Devant cette pancytopénie fébrile chez ce patient immunodéprimé, plusieurs hypothèses sont envisagées : un sepsis à point de départ cutané d'étiologie bactérienne ou fongique, une toxicité hématologique des immunosuppresseurs et, devant la splénomégalie, un SAM. Un complément biologique montre une hyperferritinémie à $13067 \mu \mathrm{g} / \mathrm{l}(32 \mathrm{~N})$ ainsi qu'une hypertriglycéridémie à $4,62 \mathrm{mmol} / \mathrm{l}(3 \mathrm{~N})$ qui viennent renforcer l'hypothèse de SAM. Le diagnostic est confirmé par le myélogramme qui montre des images d'hémophagocytose. Les charges virales dans le plasma étant positives pour le CMV inférieur à 

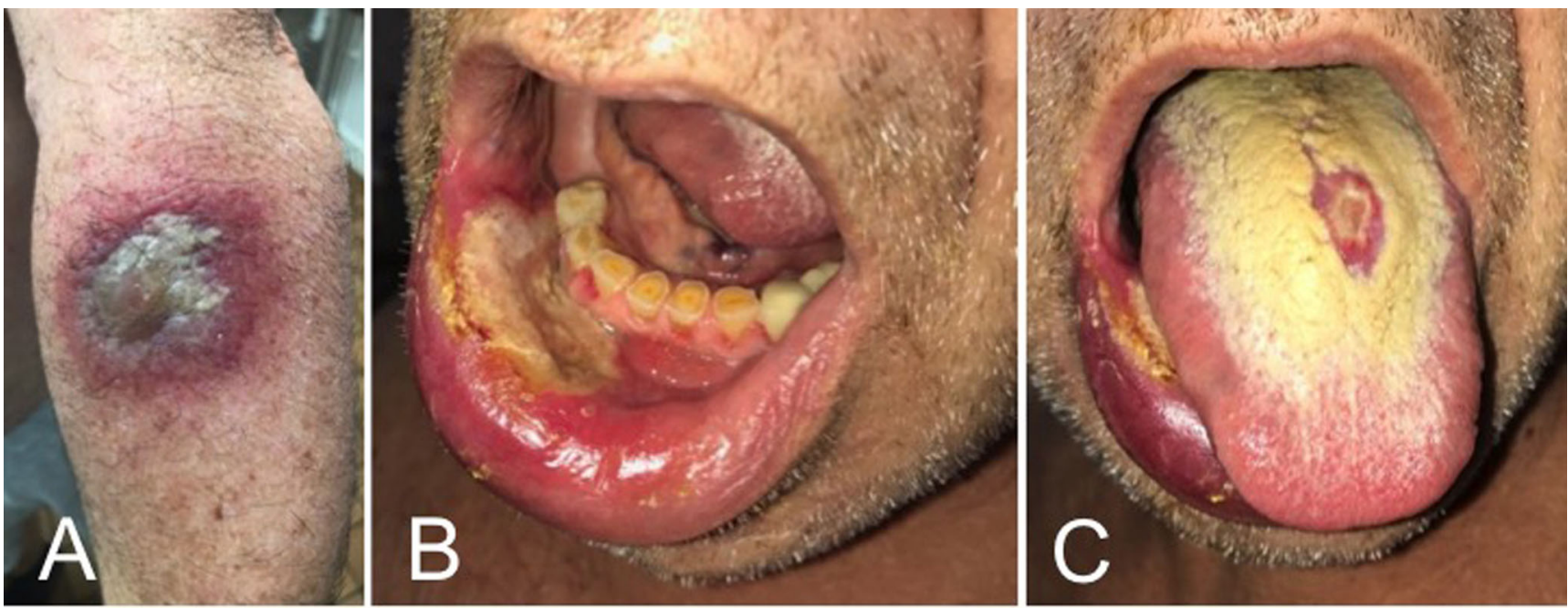

Fig. 1 Lésions cutanées à type de lésion abcédée à centre bulleux, nécrotique de la partie antérieure du bras gauche (A), d'ulcération à fond fibrineux sale de $3 \mathrm{~cm}$ de grand axe de la partie inférieure gauche de la lèvre (B) et lésion ulcérée de la langue (C)

$1,79 \log$ et pour Epstein-Barr virus (EBV) inférieur à 2,18 Log, une recherche de virus sur les prélèvements et les biopsies des lésions cutanées a été effectuée et s'est avérée négative. Les prélèvements des ulcérations cutanées objectivent du Pseudomonas aeruginosa, et l'évolution clinique et biologique du patient est favorable sous un traitement antibiotique adapté par pipéracilline-tazobactam. L'absence d'adénopathies profondes sur un scanner thoracoabdominopelvien et la normalisation des LDH ont écarté l'hypothèse d'un lymphome sous-jacent.

\section{Discussion}

Le SAM est une pathologie rare, mais dont la prévalence est probablement sous-estimée [1]. Il s'agit d'une urgence pouvant engager le pronostic vital, avec une mortalité élevée variant de 22 à $59 \%$ selon les études [2]. Ainsi, sa reconnaissance précoce par les urgentistes est primordiale, car son pronostic est essentiellement dominé par la rapidité d'instauration du traitement spécifique et étiologique.

Ce syndrome résulte d'un défaut d'activité cytotoxique des lymphocytes natural killer (NK) et des lymphocytes T$\mathrm{CD} 8+$. Au cours de la réponse immunitaire physiologique, ces cellules cytotoxiques vont détruire la cellule cible pathogène infectieuse ou cancéreuse en activant les macrophages par le biais de cytokines pro-inflammatoires. Une fois la cellule cible détruite, la stimulation antigénique s'arrête et la réaction immunitaire prend fin. Dans le SAM, les cellules cytotoxiques prolifèrent et libèrent des quantités accrues de cytokines pro-inflammatoires mais ne parviennent plus à détruire la cellule cible. Dès lors, la stimulation antigénique persiste et entretient la cascade inflammatoire entraînant un orage cytokinique.

Le SAM survient sur un terrain immunodéprimé dans plus de $80 \%$ des cas [3] (virus de l'immunodéficience humaine [VIH], traitement immunosuppresseur, transplantation de cellules souches hématopoḯtiques, hémopathie maligne) à la suite d'un facteur déclenchant. Ce facteur déclenchant est majoritairement lié à une infection ou à une hémopathie maligne (les maladies auto-immunes sontelles beaucoup plus rares ?) [4]. L'agent infectieux, isolé dans $70 \%$ des cas, est le plus fréquemment viral (EBV, VIH, HSV, CMV), parfois bactérien (essentiellement par Mycobacterium tuberculosis) et plus rarement parasitaire ou fongique, tandis que les hémopathies malignes sont dominées par les lymphomes [3]. En 2004, une étude française a rapporté, dans la plus importante série de cas de SAM survenant chez les patients greffés rénaux, que l'étiologie la plus fréquemment retrouvée était une infection virale, notamment par CMV et EBV (trois patients respectivement sur les 17 cas de SAM décrits, dont une primo-infection dans chaque cas) [5].

Le diagnostic est difficile et repose sur l'association de signes cliniques, biologiques et histologiques. Les signes cliniques majeurs associent une fièvre prolongée et intense liée à l'orage cytokinique et un syndrome tumoral (splénomégalie, hépatomégalie, adénopathies) lié à l'infiltration tissulaire secondaire à la prolifération lymphocytaire et macrophagique [6]. Une atteinte cutanée (rash aspécifique) ou une atteinte neurologique (déficit focal, confusion, convulsions, coma) sont plus rares mais parfois décrites. Les cytopénies sont un élément caractéristique du syndrome et touchent de manière variable les trois lignées. Elles sont liées à la phagocytose des éléments figurés du sang par les macrophages. 
L'hyperferritinémie, liée à la sécrétion lipidique hépatique stimulée par TNF-alpha, et l'hypertriglycéridémie, secondaire à l'hyperstimulation macrophagique, sont quasiment constantes (entre 60 et $70 \%$ des cas) [2,6,7]. Une anomalie du bilan hépatique, une hypofibrinogénémie et une augmentation des LDH sont fréquentes. La recherche d'hémophagocytose médullaire sur le myélogramme est l'examen de référence, mais cette confirmation n'est pas retenue par tous auteurs, car il s'agit d'un examen peu sensible et peu spécifique [6]. En effet, l'hémophagocytose peut être absente initialement et peut également se rencontrer au cours d'autres affections, comme lors d'un choc septique où elle est retrouvée chez environ $4 \%$ des patients [8].

Le SAM peut se compliquer d'une atteinte multiviscérale nécessitant une prise en charge en soins intensifs. Les causes d'admissions en soins intensifs décrites sont une atteinte respiratoire aiguë $(30,3 \%)$, une atteinte neurologique avec un coma ou une confusion $(21,4 \%)$, un choc $(17,8 \%)$, une insuffisance rénale aiguë $(16,1 \%)$, une hépatite fulminante (7,1\%) et un syndrome hémorragique (5,3\%) [1]. L'atteinte respiratoire aiguë est en lien avec une infiltration pulmonaire interstitielle et alvéolaire diffuse, un épanchement pleural ou des adénopathies médiastinales [9]. Les principales causes d'insuffisance rénale aiguë sont l'hypoperfusion et la nécrose tubulaire aiguë ou le syndrome de lyse tumorale [10]. À six mois, un tiers de ces patients ayant présenté une insuffisance rénale aiguë développent une maladie rénale chronique. Les troubles de la coagulation décrits en soins intensifs sont une thrombopénie, une hypofibrinogénémie, une coagulation intravasculaire disséminée chez la moitié des patients et des taux de prothrombine et facteur $\mathrm{V}$ effondrés, entraînant des complications hémorragiques graves dans $22 \%$ des cas [11]. Un SAM sévère peut avoir une présentation similaire à celle d'un choc septique, qui en est le principal diagnostic différentiel. De plus, un SAM peut se compliquer d'un choc septique ou en être la conséquence [8]. Ainsi, l'évoquer chez les patients présentant une atteinte multiviscérale atypique et inexpliquée devrait être systématique [2], étant donné la possibilité d'instaurer un traitement spécifique.

Afin d'en faciliter le diagnostic, deux scores ont été développés. Premièrement, l'Histiocyte Society a proposé en 1991 des critères diagnostiques, actualisés en 2004, dont la spécificité est de $96 \%$ et la sensibilité de 56,6\% [7]. Bien que développé à partir de données pédiatriques, ce score est souvent extrapolé chez l'adulte. Deuxièmement, le HScore a été élaboré en 2014 par un consensus d'experts à partir d'une étude rétrospective multicentrique portant sur 312 patients dans trois hôpitaux français [12]. Ce score varie de 0 à 337 points et est corrélé à la probabilité du patient de présenter un SAM (Tableau 1). Le Hscore est disponible gratuitement en ligne sur le site de l'hôpital Saint-Antoine. Chez notre patient, le HScore était respectivement de 258 avant et de
293 points après réalisation du myélogramme, soit une probabilité de SAM évaluée à plus de $99 \%$ dans les deux cas.

La prise en charge du SAM comprend trois volets. Premièrement, un traitement symptomatique : transfusions selon la profondeur des cytopénies, équilibre hydroélectrolytique, suppléance des défaillances d'organe. Deuxièmement, un traitement du facteur déclenchant : traitement anti-infectieux spécifique de l'agent causal, chimiothérapie dans le cas d'une étiologie néoplasique. Troisièmement, un traitement spécifique peut être utilisé pour tenter de contrôler l'orage cytokinique. Il n'existe pas à l'heure actuelle de recommandation sur les indications et les traitements spécifiques à utiliser, mais les experts s'accordent à préconiser un traitement par corticoïdes et/ou étoposide dans les cas où le facteur déclenchant n'est pas retrouvé ou que l'atteinte est sévère malgré le traitement étiologique mis en place [7]. Ces décisions thérapeutiques initiales doivent être multidisciplinaires et concertées.

Tableau 1 HScore évaluant la probabilité d'avoir un syndrome d'activation macrophagique. Un seuil à 169 points correspond à une sensibilité de $93 \%$ et une spécificité de $86 \%$. La probabilité est inférieure à $1 \%$ lorsque le score est inférieur ou égal à 90 points et supérieure à $99 \%$ s'il est supérieur ou égal à 250 points. D'après Fardet et al. [12]

\begin{tabular}{|c|c|}
\hline Caractéristiques & Points \\
\hline \multicolumn{2}{|l|}{ Critères cliniques } \\
\hline Immunosuppression connue & 18 \\
\hline \multicolumn{2}{|l|}{ Température maximale } \\
\hline $38,4-39,4^{\circ} \mathrm{C}$ & 33 \\
\hline$>39,4{ }^{\circ} \mathrm{C}$ & 49 \\
\hline \multicolumn{2}{|l|}{ Organomégalie } \\
\hline Hépatomégalie ou splénomégalie & 23 \\
\hline Hépatomégalie et splénomégalie & 38 \\
\hline \multicolumn{2}{|l|}{ Critères biologiques } \\
\hline \multicolumn{2}{|l|}{ Cytopénie $^{\mathrm{a}}$} \\
\hline 2 lignées & 24 \\
\hline 3 lignées & 34 \\
\hline \multicolumn{2}{|l|}{ Hyperferritinémie } \\
\hline $2000-6000 \mu \mathrm{g} / 1$ & 35 \\
\hline$>6000 \mu \mathrm{g} / 1$ & 50 \\
\hline ASAT > $30 \mathrm{UI} / 1$ & 19 \\
\hline \multicolumn{2}{|l|}{ Hypertriglycéridémie } \\
\hline $1,5-4 \mathrm{mmol} / 1$ & 44 \\
\hline$>4 \mathrm{mmol} / \mathrm{l}$ & 64 \\
\hline Hypofibrinogénémie $<2,5 \mathrm{~g} / 1$ & 30 \\
\hline \multicolumn{2}{|l|}{ Critère histologique } \\
\hline Hémophagocytose médullaire & 35 \\
\hline
\end{tabular}


Cette prise en charge par soins de soutien agressifs et précoces ainsi qu'un traitement spécifique assurent la survie de près de la moitié des patients présentant des défaillances multiviscérales, admis en soins intensifs [1].

En conclusion, le SAM est une pathologie rare pouvant potentiellement engager le pronostic vital. Son diagnostic est difficile, ce d'autant plus qu'il peut mimer un sepsis. Il faut l'évoquer devant une cytopénie fébrile avec splénomégalie et atteinte multiviscérale chez un patient immunodéprimé. La présence d'une hyperferritinémie et d'une hypertriglycéridémie est fortement évocatrice. Le recours aux différents scores peut être une aide au diagnostic. Son étiologie est principalement infectieuse ou néoplasique, parfois autoimmune. Son traitement doit être agressif et sans délai.

Liens d'intérêts : les auteurs déclarent ne pas avoir de liens d'intérêts.

\section{Références}

1. Buyse S, Teixeira L, Galicier L, et al (2010) Critical care management of patients with hemophagocytic lymphohistiocytosis. Intensive Care Med 36:1695-702

2. Créput C, Galicier L, Buyse S, Azoulay E (2008) Understanding organ dysfunction in hemophagocytic lymphohistiocytosis. Intensive Care Med 34:1177-87
3. Lerolle N, Laanani M, Rivière S, et al (2016) Diversity and combinations of infectious agents in 38 adults with an infectiontriggered reactive haemophagocytic syndrome: a multicenter study. Clin Microbiol Infect 22:268.e1-8

4. Ramos-Casals M, Brito-Zerón P, López-Guillermo A, et al (2014) Adult haemophagocytic syndrome. Lancet 383:1503-16

5. Karras A, Thervet E, Legendre C, Groupe coopératif de transplantation d'Île-de-France (2004) Hemophagocytic syndrome in renal transplant recipents: reports of 17 cases and review of literature. Transplantation 77:238-43

6. Gonzalez F, Vincent F, Cohen Y (2009) Syndrome d'activation macrophagique d'origine infectieuse : étiologies et prise en charge. Réanimation 18:284-90

7. Novotny F, Simonetta F, Samii K, et al (2017) Syndrome hémophagocytaire réactionnel. Rev Med Suisse 13:1797-803

8. Besset S, Schnell D, Azoulay E (2012) Hemophagocytic lymphohistiocytosis mimicking septic shock. Chest 141:835

9. Seguin A, Galicier L, Boutboul D, et al (2016) Pulmonary involvement in patients with hemophagocytic lymphohistiocytosis. Chest 149:1294-301

10. Aulagnon F, Lapidus N, Canet E, et al (2015) Acute kidney injury in adults with hemophagocytic lymphohistiocytosis. Am J Kidney Dis 65:851-9

11. Valade S, Azoulay E, Galicier L, et al (2015) Coagulation disorders and bleedings in critically ill patients with hemophagocytic lymphohistiocytosis. Medicine 94:1692

12. Fardet L, Galicier L, Lambotte O, et al (2014) Development and validation of the HScore, a score for the diagnosis of reactive hemophagocytic syndrome. Arthritis Rheum 66:2613-20 